\title{
Análise dos sintomas, sinais clínicos e suporte de oxigênio em pacientes com bronquiolite antes e após fisioterapia respiratória durante a internação hospitalar
}

\author{
Analysis of symptoms, clinical signs and oxygen support in patients with bronchiolitis before and after \\ chest physiotherapy during hospitalization
}

Giselle de Castro ${ }^{1}$, Renata Remondini², Adriana Zamprônio dos Santos ${ }^{3}$, Cristiane do Prado 4

\section{RESUMO}

Objetivo: Avaliar os efeitos da fisioterapia em pacientes pediátricos, internados com bronquiolite.

Métodos: O estudo incluiu 29 pacientes menores de um ano, com diagnóstico médico de bronquiolite aguda, sem cardiopatia congênita não corrigida, neuropatia, doença pulmonar de base ou que necessitassem de suporte ventilatório, no período de março a julho de 2009. Foi avaliada, por meio de questionário, a opinião dos pais ou responsáveis acerca das condições clínicas do paciente antes e após a primeira sessão de fisioterapia. Foram realizadas manobras de desobstrução brônquica, como drenagem postural, tapotagem, vibração, aceleração de fluxo expiratório e aspiração nasotraqueal. A avaliação foi realizada diariamente por meio de exame físico antes do atendimento fisioterapêutico e 15 a 45 minutos após, seguindo uma ficha específica. Os desfechos analisados foram: modificações do suporte de oxigênio, sinais clínicos (ausculta pulmonar, presença de tiragens, oxigenação e classificação da gravidade pelo escore de Downes) e sintomas (inapetência, hipoatividade, dificuldade para dormir, obstrução nasal e tosse constante).

Resultados: Dos 29 pacientes avaliados, houve melhora significativa nos seguintes sinais e sintomas: ausculta pul- monar com ruídos adventícios $(p<0,001)$, estertores subcrepitantes $(p=0,017)$ e sibilos $(p=0,010)$, tiragens de modo geral $(p<0,001)$, tiragem intercostal $(p<0,001)$ e classificação da gravidade pelo escore de Downes $(p<0,001)$. Também foi observada melhora significativa em todos os aspectos avaliados no questionário $(p<0,001)$.

Conclusões: A fisioterapia respiratória promoveu uma melhora significante em curto prazo das condições clínicas dos pacientes com bronquiolite aguda.

Palavras-chave: bronquiolite; serviço hospitalar de fisioterapia; pediatria.

\section{ABSTRACT}

Objective: To evaluate the effects of chest physiotherapy in hospitalized patients with bronchiolitis.

Methods: The study included 29 patients younger than one year, diagnosed with acute bronchiolitis, without uncorrected congenital heart disease, neuropathy, lung pathology or need of mechanical ventilation, from March to July 2009. The opinion of the parents or guardians was evaluated using a questionnaire about the clinical condition of the patient
Instituição: Departamento Materno-Infantil do Hospital Israelita Albert Einstein (HIAE), São Paulo, SP, Brasil

${ }^{1}$ Especialista em Fisioterapia Respiratória Pediátrica pelo Instituto da Criança do Hospital das Clínicas da Faculdade de Medicina da Universidade de São Paulo (USP); Fisioterapeuta do Departamento Materno-Infantil do HIAE, São Paulo, SP, Brasil

${ }^{2}$ Especialista em Fisioterapia Cardiorrespiratória pelo Instituto do Coração do Hospital das Clínicas da Faculdade de Medicina da USP; Fisioterapeuta do Departamento Materno-Infantil do HIAE, São Paulo, SP, Brasil

${ }^{3}$ Especialista em Fisioterapia Respiratória pela Universidade Adventista de São Paulo (Unasp); Fisioterapeuta do Departamento Materno-Infantil do HIAE, São Paulo, SP, Brasil

${ }^{4}$ Especialista em Fisioterapia Respiratória e Fisiologia do Exercício pela Universidade Federal de São Paulo (Unifesp); Coordenadora do Serviço de Fisioterapia do Departamento Materno-Infantil do HIAE, São Paulo, SP, Brasil
Endereço para correspondência:

Renata Remondini

Rua Inácio, 380 - Vila Zelina

CEP 03142-000 - São Paulo/SP

E-mail: rremondini@yahoo.com.br

Conflito de interesse: nada a declarar

Recebido em: 28/9/2010

Aprovado em: 4/4/2011 
before and after the first session of chest physiotherapy. Airway clearance techniques, such as postural drainage, manual percussion, vibration, acceleration of expiratory flow and tracheal suction were applied. The evaluation was performed daily by physical examination prior to physiotherapy and 15 to 45 minutes later, following a specific form. The outcomes observed were: changes of oxygen support, clinical signs (pulmonary auscultation, presence of retractions, oxygenation and severity classification by Downes score) and symptoms (difficulty in sleeping and in feeding, decreased activities, nasal obstruction and constant cough).

Results: Among the 29 patients evaluated, a significant improvement was noticed in the following signs and symptoms: pulmonary auscultation with adventitious sounds general $(p<0.001)$, rales $(p=0.017)$ and wheezes $(p=0.010)$; retractions - general $(p<0.001)$ and intercostal retraction $(p<0.001)$; severity of Downes Score $(p<0.001)$. A significant improvement was also noted in all aspects evaluated in the questionnaire $(p<0.001)$.

Conclusions: Chest physiotherapy lead to a significant short term improvement in infants with bronchiolitis.

Key-words: bronchiolitis; physical therapy department, hospital; pediatrics.

\section{Introdução}

A bronquiolite aguda é uma das doenças mais frequentes do sistema ventilatório e acomete neonatos, lactentes e crianças até os três anos de idade, com predominância nos seis primeiros meses de vida, afetando mais os recém-nascidos prematuros, e leva, em muitos casos, à necessidade de internação hospitalar. Possui característica sazonal, ocorrendo epidemicamente nos meses de outono e inverno. As infecções respiratórias agudas são importantes causas de morbimortalidade, particularmente nos países em desenvolvimento ${ }^{(1-3)}$.

A bronquiolite aguda pode ser caracterizada por diversas manifestações clínicas, como coriza, febre, tosse e sibilância ${ }^{(4)}$. A radiografia de tórax é caracterizada por hiperinsuflação, infiltrados grosseiros e preenchimento peribrônquico ${ }^{(1)}$. Algumas escalas podem avaliar a gravidade da bronquiolite apor meio da análise de variáveis clínicas, como a escala Wood-Downes modificada por Ferres, a qual caracteriza a doença em leve (1 a 3 pontos), moderada ( 4 a 7 pontos) ou grave ( 8 a 14 pontos). Os itens analisados (sibilos, tiragem, frequência respiratória, frequência cardíaca, ventilação e cianose) recebem pontuações de 0 a 3, dependendo da presença e da intensidade dos mesmos ${ }^{(5)}$.
O tratamento da bronquiolite aguda ainda é controverso. A terapêutica inclui hidratação, oxigenação, fisioterapia respiratória e medicamentos, como broncodilatadores, adrenalina, mucolíticos e corticoide inalatório ${ }^{(1,6,7)}$. A fisioterapia respiratória tem sido utilizada em pacientes com bronquiolite aguda com os objetivos de desobstrução brônquica, desinsuflação pulmonar e recrutamento alveolar, por meio de diversas técnicas, como posicionamento, aumento do fluxo expiratório (AFE), vibração manual, tapotagem e aspiração das vias aéreas ${ }^{(8,9)}$. Em três estudos foram relatados efeitos benéficos dessas técnicas em pacientes com bronquiolite aguda ${ }^{(1,2)}$.

Crianças hospitalizadas por bronquiolite apresentam um risco elevado de sequela pulmonar, caracterizada principalmente por tosse e sibilância recorrente, podendo acarretar um maior número de visitas em pronto-atendimento e, até mesmo, reinternações ${ }^{(4)}$. Alguns autores não indicam a fisioterapia respiratória na fase aguda da bronquiolite devido ao fato de as manobras de higiene brônquica causarem agitação na criança, aumentando, assim, a hipoxemia e, eventualmente, o broncoespasmo ${ }^{(10,11)}$. Há até relato de fratura de costelas devido à intensidade das manobras de higiene brônquica ${ }^{(12)}$. Já outros autores acreditam que a fisioterapia respiratória seja benéfica por promover diminuição do tempo de internação hospitalar e evitar a necessidade de suporte ventilatório ${ }^{(2,7)}$.

É importante analisar a intervenção fisioterapêutica em lactentes com bronquiolite, utilizando técnicas adequadas de acordo com a idade e a colaboração do paciente durante o atendimento. As orientações são fundamentais para uma boa evolução durante a instalação do quadro em relação ao posicionamento adequado e estimulação para eliminar as secreções. Dessa forma, faz-se necessário investigar os efeitos da fisioterapia respiratória em pacientes com bronquiolite aguda. $\mathrm{O}$ presente estudo teve como objetivo avaliar os efeitos da fisioterapia respiratória em recém-nascidos e lactentes com diagnóstico de bronquiolite aguda durante a internação hospitalar antes e após a intervenção fisioterapêutica de AFE, tapotagem, vibração manual, aspiração nasotraqueal e posicionamento. Os desfechos analisados foram as modificações dos parâmetros cardiorrespiratórios (suporte de oxigênio, ausculta pulmonar, presença de tiragens, saturação periférica de oxigênio e escore de Downes) e identificação das opiniões dos pais ou responsáveis quanto à fisioterapia.

\section{Método}

Neste estudo, foram avaliados recém-nascidos e lactentes com diagnóstico de bronquiolite aguda, internados na ala pediátrica, semi-intensiva pediátrica e Unidade de Terapia 
Intensiva Pediátrica (UTIP) de um hospital particular, no período de março a julho de 2009. Foram selecionados pacientes com idade até um ano, de ambos os sexos e com diagnóstico de bronquiolite aguda. Não foram incluídos pacientes portadores de cardiopatia congênita não corrigida, neuropatia, alguma doença pulmonar de base ou que necessitassem de suporte ventilatório.

A avaliação foi realizada diariamente, por meio do exame físico, antes do atendimento fisioterapêutico e 15 a 45 minutos após, seguindo uma ficha de avaliação proposta, na qual foram anotados os dados relativos à oxigenoterapia, ausculta pulmonar com ruídos adventícios, estertores subcrepitantes, estertores crepitantes, sibilos e roncos, presença de tiragens geral, batimento de asa de nariz, tiragem intercostal, tiragem subdiafragmática e tiragem esternal, além da saturação periférica de oxigênio $\left(\mathrm{SpO}_{2}\right)$ e classificação da bronquiolite pelo escore de Downes. A primeira avaliação, antes do atendimento fisioterapêutico, foi feita pelo profissional que realizou o mesmo. Já a segunda parte da avaliação, 15 a 45 minutos após o término do atendimento, foi feita por um segundo fisioterapeuta para evitar possíveis vieses na análise da resposta.

$\mathrm{O}$ atendimento fisioterapêutico foi constituído por posicionamento, AFE, tapotagem, vibração manual e aspiração nasotraqueal, com duração de, aproximadamente, dez minutos cada técnica (duração da terapia de 40 a 50 minutos), conforme a necessidade do paciente. $\mathrm{O}$ número de atendimentos foi realizado de acordo com o quadro clínico de cada criança e dias de internação hospitalar.

Antes e após a primeira sessão de fisioterapia respiratória, foi entregue, pelo paciente, aos pais ou responsável, um questionário, o qual avaliou os efeitos da terapia no paciente em curto prazo, por meio das seguintes questões de múltipla escolha: "A criança apresenta dificuldade para dormir?", "A criança apresenta inapetência?”, "A criança apresenta hipoatividade?”, “A criança apresenta obstrução nasal?” e "A criança apresenta tosse constantemente?”, com alternativas "Sim" e "Não" para cada questão.

O acompanhamento do paciente foi realizado após autorização de seus pais ou responsável, assinando um termo de consentimento informado. Os procedimentos realizados neste estudo foram aprovados pelo Comitê de Ética em Pesquisa do Hospital Israelita Albert Einstein.

A idade dos pacientes foi descrita por média, desvio padrão, mínimo e máximo, e o gênero, por frequências absolutas. Os sinais e sintomas avaliados antes e após o atendimento fisioterapêutico foram descritos por frequências absolutas e porcentagens, assim como os itens do questionário. A comparação entre sinais e sintomas nos instantes pré e pós foi realizada utilizando modelos de equações de estimação generalizada $(E E G)^{(13)}$, de modo a contemplar a dependência entre os atendimentos realizados em um mesmo paciente. Em relação aos itens do questionário, a comparação foi feita com o uso do teste de McNemar. Todas as análises foram feitas considerando-se o nível de significância de $5 \%$ e utilizando o programa estatístico Statistical Package for the Social Science (SPSS), versão 17.0.

\section{Resultados}

Dos 29 pacientes, 16 eram do sexo feminino. Apenas quatro apresentavam antecedentes mórbidos (um com broncoespasmo, um com bronquiolite, um com meningite e um com prematuridade). A média de idade era de 4,6 meses (desvio padrão de três meses), variando de 16 dias a nove meses.

Nas comparações em relação aos sinais e sintomas observados nos 97 atendimentos, houve melhora significativa em seis dos 13 itens avaliados. Foram eles: ausculta pulmonar com ruídos adventícios (estertores subcreptantes e sibilos), tiragens intercostais e classificação de Downes (Gráfico 1). $\mathrm{Na}$ avaliação da ausculta pulmonar, nos 92 atendimentos em que foram observados ruídos adventícios, 13 passaram a não apresentar tais ruídos após a fisioterapia e, dos cinco que não tinham ruídos, dois evoluíram com piora. Dos 47 que apresentavam tiragens, 26 passaram a não apresentar, porém, dos 50 que não apresentavam, oito evoluíram com tiragens após o atendimento. Em relação ao escore de Downes, dos 56 que apresentavam escore leve, quatro evoluíram para moderado. Escore moderado foi observado em 40 atendimentos, dentre os quais 20 evoluíram para leve. O único atendimento no qual o paciente apresentava escore grave antes da fisioterapia evoluiu para leve após a mesma (Tabela 1).

Em relação à oxigenoterapia, dos 21 pacientes que a utilizavam, sete passaram a não utilizar e, dos 76 que não utilizavam, três evoluíram com necessidade de $\mathrm{O}_{2}$. Dos nove atendimentos nos quais foi observada oxigenação desfavorável (considerando-se ideal $\mathrm{SpO}_{2} \geq 93 \%$ ), seis evoluíram com melhora, entretanto, dos 88 que apresentavam $\mathrm{SpO}_{2}$ ideal, nove evoluíram com piora. Apesar das mudanças observadas, não houve diferença estatisticamente significante em relação à oxigenoterapia e $\mathrm{SpO}_{2}$ antes e após os atendimentos.

Os cinco sintomas avaliados no questionário estavam presentes na maioria dos pacientes do estudo. Pode-se observar, no Gráfico 2, melhora em todos os itens, com redução significativa no número de pacientes com sintomas. $\mathrm{O}$ item 


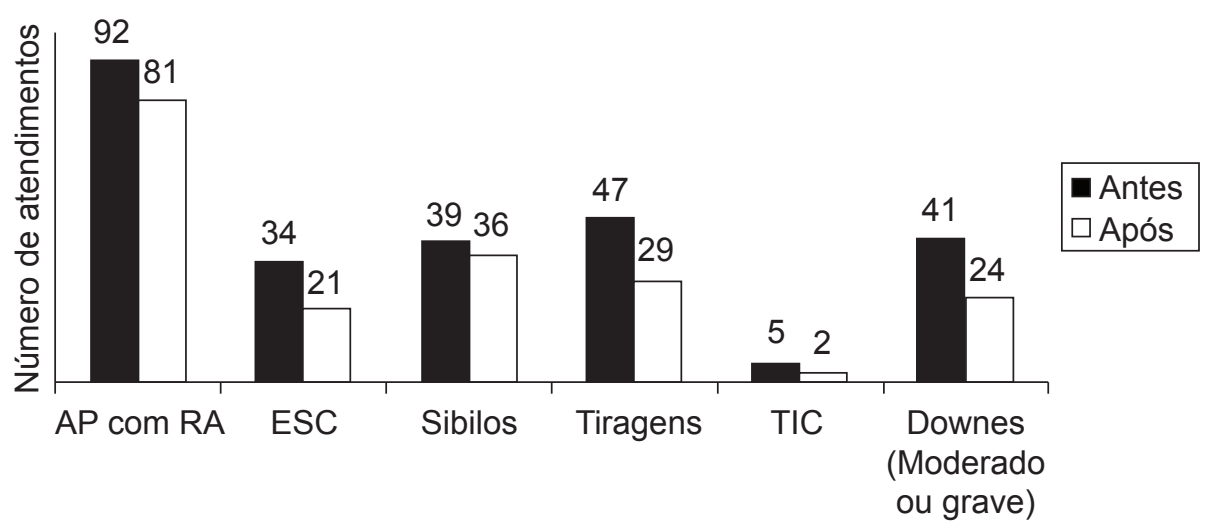

AP com RA: ausculta pulmonar com ruídos adventícios; ESC: estertores subcrepitantes; TIC: tiragem intercostal.

Gráfico 1 - Comparação dos sinais antes e após o atendimento fisioterapêutico - itens com significância estatística (total de 97 atendimentos)

Tabela 1 - Comparação dos sinais e sintomas antes e após o atendimento fisioterapêutico

\begin{tabular}{|c|c|c|c|c|c|}
\hline \multirow{2}{*}{ Item } & \multicolumn{2}{|c|}{ Não mudaram } & \multicolumn{2}{|c|}{ Mudaram } & \multirow[b]{2}{*}{ Valor $p$} \\
\hline & Não/não (\%) & Sim $/ \operatorname{sim}(\%)$ & Pioraram (\%) & Melhoraram (\%) & \\
\hline Oxigenioterapia & $73(75,3)$ & $14(14,4)$ & $3(3,1)$ & $7(7,2)$ & 0,585 \\
\hline AP com RA & $3(3,1)$ & $79(81,4)$ & $2(2,1)$ & $13(13,4)$ & $<0,001$ \\
\hline Estertores subcrepitantes & $36(45,6)$ & $12(15,27)$ & $9(11,4)$ & $22(22,8)$ & 0,017 \\
\hline Estertores crepitantes & $57(72,2)$ & $1(1,3)$ & $18(22,8)$ & $3(3,8)$ & 0,121 \\
\hline Sibilos & $30(38,0)$ & $26(32,9)$ & $10(12,7)$ & $13(16,5)$ & 0,010 \\
\hline Roncos & $31(39,2)$ & $18(22,8)$ & $5(6,3)$ & $25(31,6)$ & 0,210 \\
\hline Tiragens & $42(43,3)$ & $21(21,6)$ & $8(8,2)$ & $26(26,8)$ & $<0,001$ \\
\hline Batimento de asa nasal & $21(100)$ & 0 & 0 & 0 & - \\
\hline Tiragem intercostal & $15(71,4)$ & $1(4,8)$ & $1(4,8)$ & $4(19,0)$ & $<0,001$ \\
\hline Tiragem subdiafragmática & 0 & $18(85,7)$ & $2(9,5)$ & $1(4,8)$ & 0,277 \\
\hline Tiragem esternal & $20(95,2)$ & 0 & 0 & $1(4,8)$ & $>0,999$ \\
\hline $\mathrm{SpO}_{2} \geq 93 \%$ & $3(3,1)$ & $79(81,4)$ & $6(6,2)$ & $9(9,3)$ & 0,610 \\
\hline Downes moderado/grave** & $52(53,6)$ & $20(20,6)$ & $4(4,1)$ & $21(21,6)$ & $<0,001$ \\
\hline
\end{tabular}

Porcentagens em relação ao total de 97 atendimentos; ${ }^{* *}$ não/não=leve no pré e no pós; sim/sim=moderado ou grave no pré e no pós. AP com RA: ausculta pulmonar com ruídos adventícios.

em que houve melhora mais evidente foi "dificuldade para dormir" (dos 26 pacientes que apresentavam o sintoma antes da fisioterapia, somente seis permaneceram com essa dificuldade após a fisioterapia). Chama atenção o item "tosse constante", que, apesar de apresentar o segundo maior percentual de pacientes que melhoraram, foi o único em que se observou piora (Tabela 2), embora isso tenha ocorrido em apenas dois pacientes.

\section{Discussão}

As características fisiopatológicas da doença correspondem ao acúmulo de secreções nas vias aéreas, obstrução e colapso de unidades alveolares e hiperinsuflação pulmonar ${ }^{(1)}$. Lactentes com bronquiolite viral aguda apresentam ruídos adventícios na ausculta pulmonar devido ao processo inflamatório nas vias aéreas inferiores, assim como acúmulo de secreção e material desprendido da mucosa respiratória ${ }^{(14)}$. Manifestações clínicas como taquipneia, hipóxia leve a moderada e sinais de desconforto respiratório (batimento de asa de nariz e retrações da musculatura acessória da respiração - intercostais e subcostais) são frequentes em pacientes com bronquiolite viral aguda. Também podem estar presentes tosse, febre, coriza, ruídos adventícios na ausculta pulmonar (sibilos, crepitações ou roncos), expansão torácica diminuída e fase expiratória prolongada ${ }^{(1,15,16)}$. 


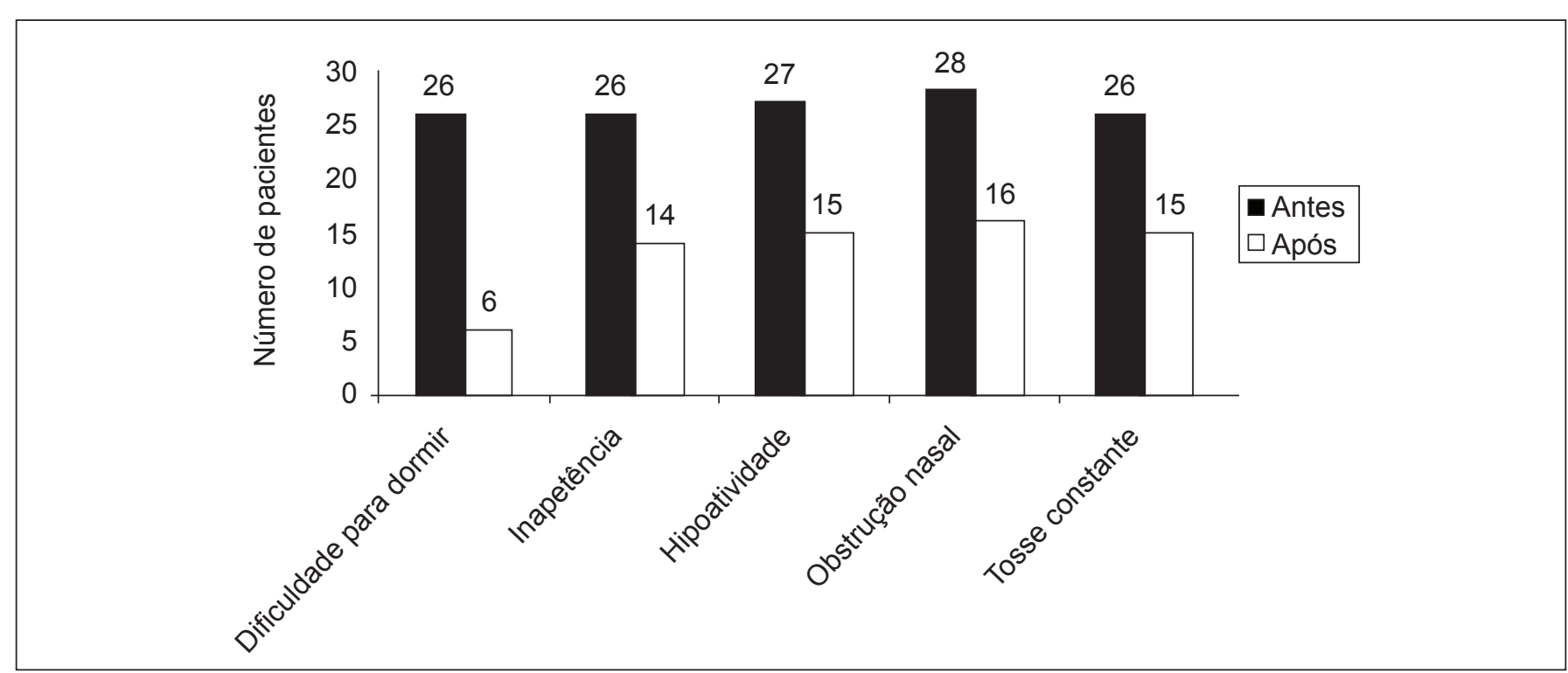

Gráfico 2 - Análise do questionamento sobre as atividades antes e após a fisioterapia - itens com significância estatística (total de 29 pacientes).

Tabela 2 - Análise do questionário sobre o estado clínico do paciente antes e após a fisioterapia no primeiro atendimento

\begin{tabular}{lcccccc}
\hline & & \multicolumn{2}{c}{ Não mudaram } & \multicolumn{2}{c}{ Mudaram } & Valor $\boldsymbol{p}$ \\
\cline { 3 - 6 } Item & $\mathbf{n}$ & Não/não (\%) & Sim/sim (\%) & Pioraram (\%) & Melhoraram (\%) & Val \\
Dificuldade para dormir & 29 & $3(10,3)$ & $6(20,7)$ & - & $20(69,0)$ & $<0,001$ \\
Inapetência & 29 & $3(10,3)$ & $14(48,3)$ & - & $12(41,4)$ & $<0,001$ \\
Hipoatividade & 29 & $2(6,9)$ & $15(51,7)$ & - & $12(41,4)$ & $<0,001$ \\
Obstrução nasal & 29 & $1(3,4)$ & $16(55,2)$ & - & $12(41,4)$ & $<0,001$ \\
Tosse constante & 29 & $1(3,4)$ & $13(44,8)$ & $2(6,9)$ & $13(44,8)$ & 0,007 \\
\hline
\end{tabular}

Diante desse contexto, optou-se por realizar um estudo referente a esse tratamento, a fim de confirmar ou não os achados obtidos na literatura.

Neste estudo, observou-se melhora significante quanto à presença de tiragens após a fisioterapia, principalmente tiragens intercostais. Em relação à tiragem subdiafragmática, em dois atendimentos os pacientes não a apresentavam antes da fisioterapia, porém evoluíram com esse tipo de tiragem após a terapia. A Academia Americana de Pediatria ${ }^{(17)}$, em revisão de estudos clínicos randomizados, de coorte e caso-controle encontrados na literatura, desenvolveu níveis de recomendação para os recursos terapêuticos aplicados na bronquiolite e concluiu que a fisioterapia respiratória não deve ser usada rotineiramente, pois eleva o nível de estresse na criança. No entanto, sugere-se que a fisioterapia, em crianças com bronquiolite, seja capaz de desobstruir as vias aéreas, remover secreções e prevenir atelectasias ${ }^{(16,18)}$. Segundo Carvalho et a $l^{(1)}$, a fisioterapia respiratória pode ser indicada durante todo o curso da bronquiolite por proporcionar desobstrução brônquica, desinsuflação pulmonar e recrutamento alveolar.

No presente estudo, observou-se melhora da ausculta pulmonar após a fisioterapia na maioria dos atendimentos realizados, principalmente em relação à presença de estertores subcrepitantes e sibilos. Lanza et al notaram que a aplicação de técnicas de fisioterapia respiratória em pacientes hospitalizados por bronquiolite proporcionou redução do desconforto respiratório, maior quantidade de secreção aspirada e melhora qualitativa na ausculta pulmonar após a realização de vibrocompressão e tapotagem ${ }^{(14)}$. Em um estudo com 12 lactentes com padrão respiratório obstrutivo, Postiaux ${ }^{(19)}$ também observou diminuição significante de sibilos após a inalação com broncodilatadores, principalmente quando esta se associou à execução de manobras fisioterapêuticas, já que os sibilos encontrados eram provenientes de secreção excessiva ${ }^{(16)}$. Nos estudos de Webb et al ${ }^{(20)}$, Nicholas et $a l^{(21)}$ e Bohé et $a l^{(22)}$, 
os pacientes com bronquiolite não obtiveram benefício na evolução do quadro clínico da doença com as técnicas de fisioterapia aplicadas (drenagem postural, tapotagem e vibração). Já em estudos franceses, além das técnicas de percussão e vibração, a técnica de aceleração do fluxo expiratório, em especial, mostrou-se efetiva para remover secreções, sendo a fisioterapia respiratória recomendada em um consenso para o manejo e o tratamento da doença $a^{(15,20,22)}$.

Luisi afirma que, ainda que não existam evidências diretas que demonstrem os benefícios da fisioterapia respiratória nos pacientes com bronquiolite, as técnicas de higiene brônquica e recrutamento alveolar são solicitadas para o tratamento dessa doença em serviços de várias partes do mundo. A fisioterapia respiratória tem sido utilizada para desobstrução e higiene brônquica, prevenção de atelectasias e recrutamento alveolar, pois proporciona diminuição da resistência das vias aéreas, promovendo melhor ventilação-perfusão e, consequentemente, diminuindo o trabalho ventilatório por meio da remoção do excesso de muco que se acumula nas vias aéreas ${ }^{(15)}$.

Perrotta et al ${ }^{(9,23)}$ incluíram em seu trabalho o estudo realizado por Postiaux ${ }^{(23)}$, que mostrou não haver evidência científica quanto à eficácia da fisioterapia respiratória em recém-nascidos e lactentes portadores de bronquiolite, porém, a utilização de um escore, baseado em sinais clínicos, poderia contribuir para respaldar as técnicas de desobstrução nesses pacientes. No presente estudo, utilizou-se o escore de Wood-Downes modificado por Ferrés ${ }^{(5)}$, sendo foi evidente a melhora clínica dos pacientes que apresentavam escore de Downes moderado e grave após a fisioterapia respiratória.

$\mathrm{Na}$ análise do questionário, houve melhora significante em relação aos diversos itens avaliados, incluindo "dificuldade para dormir", "inapetência", "hipoatividade", "obstrução nasal" e "tosse constante" após o atendimento de fisioterapia, pois a hipersecreção pulmonar dificultava diretamente

\section{Referências bibliográficas}

1. Carvalho WB, Johnston $C$, Fonseca MC. Bronquiolite aguda, uma revisão atualizada. Rev Assoc Med Bras 2007;53:182-8.

2. Lourenção LG, Salomão Junior JB, Rahal P, Souza FP, Zanetta DM. Infecções pelo vírus sincicial respiratório em crianças. Pulmão RJ 2005; 14:59-68.

3. Miyao CR, Gilio AE, Vieira S, Hein N, Pahl MM, Betta SL et al. Infecções virais em crianças internadas por doença aguda no trato respiratório inferior. J Pediatr (Rio J) 1999;75:334-44.

4. Albernaz EP, Menezes AM, César JA, Victora CG, Barros FC. Hospitalização por bronquiolite aguda como fator de risco para sibilância recorrente. Cad Saude Publica 2000;16:1049-57. os sintomas avaliados. Atualmente não há muitos estudos na literatura que comprovem o benefício qualitativo da fisioterapia respiratória em pacientes com bronquiolite. Em contrapartida, na prática clínica, observa-se um número crescente de prescrições de fisioterapia respiratória para lactentes com essa doença, principalmente na redução de complicações pulmonares ${ }^{(16)}$. Alguns autores referem que crianças com desconforto respiratório leve e que se alimentam normalmente devem ser apenas observadas. Já as que apresentam desconforto respiratório importante, com dificuldade para alimentação, risco de refluxo e broncoaspiração, além de maior edema de vias aéreas superiores, devem ser tratadas com hidratação e aspiração oral e nasal ${ }^{(17,18)}$. A partir deste estudo, conclui-se que a fisioterapia respiratória promoveu uma melhora significante em curto prazo em muitos sinais e sintomas em pacientes com bronquiolite.

No entanto, o estudo apresenta algumas limitações, como a falta de cálculo de poder amostral. Assim, este trabalho tem um maior valor descritivo do que inferencial, não sendo possível extrapolar os resultados obtidos. A ausência de grupo controle, como foi o caso, torna os resultados menos fidedignos; entretanto houve dificuldade em obter um grupo controle devido aos atendimentos ocorreram em um hospital no qual a maioria dos médicos prescreve fisioterapia respiratória para pacientes com bronquiolite. Acredita-se que novos estudos devam ser feitos para oferecer subsídios científicos à discussão das vantagens da fisioterapia em crianças hospitalizadas por bronquiolite.

\section{Agradecimentos}

Expressamos a nossa gratidão aos fisioterapeutas do Departamento Materno Infantil do HIAE e às pessoas que prestaram assistência relevante para a realização deste estudo.

5. Ferrés J. Comparison of two nebulized treatments in wheezing infants. Eur Respir J 1988; 1 (Suppl):306.

6. Rozov T. Doenças pulmonares em pediatria: diagnóstico e tratamento. São Paulo: Atheneu; 2005.

7. Sarmento GJ. Fisioterapia respiratória no paciente crítico - rotinas clínicas. Barueri: Manole; 2005.

8. Balachandran A, Shivbalan S, Thangavelu S. Chest physiotherapy in pediatric practice. Indian Pediatr 2005;42:559-68.

9. Perrotta C, Ortiz Z, Roqué i Figuls M. Chest physiotherapy for acute bronchiolitis in paediatric patients between 0 and 24 months old. Cochrane Database Syst Rev 2005:CD004873. 
10. Chalumeau M, Foix-L'Helias L, Scheinmann P, Zuani P, Gendrel D, Ducoule-Pointe HD. Rib fractures after chest physiotherapy for bronchiolitis or pneumonia in infants. Pediatr Radiol 2002;32:644-7.

11. Presto BL, Presto LD. Fisioterapia respiratória, uma nova visão. Rio de Janeiro: Loyola; 2003.

12. Wallis $C$, Prasad A. Who needs chest physiotherapy? Moving from anecdote to evidence. Arch Dis Child 1999;80:393-7.

13. Liang KY, Zeger SL. Longitudinal data analysis using generalized linear models. Biometrika 1986;73:13-22.

14. Lanza FC, Cadrobbi C, Gazzotti MR, Faria R, Luque A, Solé D. Fisioterapia respiratória em lactentes com bronquiolite: realizar ou não? Mundo Saude 2008;32:183-8.

15. Luisi F. O papel da fisioterapia respiratória na bronquiolite viral aguda. Sci Med 2008;18:39-44.

16. Mucciollo MH, Simionato NA, Paula LC, Feola AI, Monteiro VC, Ceccon ME. Fisioterapia respiratória nas crianças com bronquiolite viral aguda: visão crítica. Pediatria (São Paulo) 2008;30:257-64.

17. American Academy of Pediatrics. Subcommittee on Diagnosis and
Management of Bronchiolitis. Diagnosis and management of bronchiolitis. Pediatrics 2006;118:1774-93.

18. Clover RD. Clinical practice guideline for bronchiolitis: key recommendations Am Fam Physician 2007;75:171.

19. Postiaux G. Auscultation pulmonaire et kinésithérapie en pédiatre. Rev Mal Respir 1999;16 (Suppl 3):206-7.

20. Webb MS, Martin JA, Cartlidge PH, Ng YK, Wright NA. Chest physiotherapy in acute bronchiolitis. Arch Dis Child 1985;60:1078-9.

21. Nicholas KJ, Dhouieb MO, Marshall TG, Edmunds AT, Grant MB. An evaluation of chest physiotherapy in the management of acute bronchiolitis: changing clinical practice. Physiotherapy 1999;85:669-74.

22. Bohé L, Ferrero ME, Cuestas E, Polliotto L, Genoff M. Indicación de la fisioterapia respiratoria convencional en la bronchiolitis aguda. Medicina (B. Aires) 2004;64:198-200.

23. Postiaux G, Dubois R, Marchand E, Demay M, Jacquy J, Mangiaracina M Effets de la kinésithérapie respiratoire associant Expiration Lente Prolongée et Toux Provoquée dans la bronchiolite du nourrisson. Kinésither Rev 2006; 6:35-41. 